\title{
URBANIT \\ Urban Ontologies to Support Informed Urban Development and Planning
}

\author{
John Barton \\ City Futures Research Centre, Faculty of Built Environment \\ University of New South Wales, Sydney, Australia
}

\author{
Hairong $\mathrm{Yu}$ \\ School of Information Systems, Technology and Management, Australian School of Business \\ University of New South Wales, Sydney, Australia
}

\begin{abstract}
Keywords: Spatial decision support, Ontology, Urban planning, Urban modelling.
Abstract: When dealing with complex and multi-faceted urban design challenges, the sheer weight of the information available can make discerning the 'bigger picture' challenging. It is the suggestion of this paper that there is a requirement for intelligent tools and mechanisms to assist in the capturing, comprehending and communication of solutions to such problems whilst keeping in mind the consensus of the aims and targets. To make knowledgeable decisions, there is a need to access the most relevant sources of information possible. Quality intelligence requires a quality foundation of data. This paper will outline some fundaments of how to best structure urban components and then examine how these can be applied to assist in improving design and planning of urban precincts. In conclusion, some next steps are proposed for the development of these tools and their application within an urban context.
\end{abstract}

\section{INTRODUCTION}

This paper is reporting on the UrbanIT project undertaken at the City Futures Research Centre at the University of New South Wales. The project has been undertaken in partnership with the City of Sydney Council, the NSW Department of Planning and Landcom, a government development corporation. A component of this research was to investigate how the physical objects, concepts and systems that comprise urban environments can be defined and structured using an ontological, or semantic, approach.

This paper presents existing technologies used in structuring urban information, their interconnections and potential applications. We then look specifically at the Australian context regarding available government datasets and present applied examples of how these sets can be combined to present urban information for decision support.

One challenge presented is when sources are incomplete or inconsistently structured. An ontological approach has the capacity to provide a unified, connective framework.

\section{ONTOLOGIES AND URBAN PLANNING}

In computer science, an ontology is a formal explicit description of concepts in a domain of discourse (Gruber 2009). Ontologies are designedly flexible and consequently, can adapt to a particular domain's knowledge structure to serve a number of purposes. An ontology defines a common vocabulary and structure for those who need to share mutual understanding of their domain information. The process of logically ordering and organising this knowledge makes it explicit and easily communicated. Because it is structured, this can be recognised by both humans and machines. It provides the bridge between high-level human conceptualisation and low-level physical or logical database schema (Uschold and Gruninger 2004). 
Once a framework has been established, interrelationships, rules and logical rationales can support reasoning and knowledge discovery. These machineinterpretable definitions of essential concepts and relationships fulfil a great need for human-centred modelling services that are pursued by modern urban planners (Katranuschkov, Gehre et al. 2003).

An ontology adopts an open-world assumption (OWA): put simply, there is an appreciation that just because a concept might not be explicitly defined, it may be just as valid. This approach lends itself well to handling unstructured or semi-complete datasets. In our own work we have employed Protégé, an open-source knowledge-base framework with ontology editing features (Protégé, 2010). However, how the ontologies connect to existing government database systems containing real-world content becomes a pressing issue that needs technical resolution to provide a working example as described in section 5 .

\section{FRAMEWORKS FOR URBAN MODELLING}

The Semantic Web Stack, as proposed by Tim Berners-Lee (Berners-Lee, Hendler et al. 2001), represents a layer-based model that takes low-level machine data and 'humanises' it toward the higher levels. The ontologies are expressed as an intermediary layer. The technical elements in data or low-level schema can be mapped to higher concepts a person can easily understand, or in our case, share a common understanding with others about the built environment.

The Open Geospatial Consortium (OGC) specifies the standards and protocols to facilitate geospatial web-based services. The 3D Information Management (3DIM) working group is concerned with developing the technologies for 3D urban environments:

"A framework of data interoperability should exist across the lifecycle of building and infrastructure investment: planning, design, construction, operation, and decommissioning. This work is of interest to the geospatial community in that there is a growing need for technologies and information to effectively interoperate between these domains to support a range of vital services and decision support needs"

(www.opengeospatial.org/projects/groups/ 3dimwg)

The OGC Web Services Phase 4 testbed was conducted in 2006 to extend and demonstrate the geospatial interoperability of urban modelling technologies. This resulted in the development of several new components that demonstrate the integration of Building Information Modelling (BIM) with the OGC Architecture. With increased uptake and development of the semantic content of information repositories, there is a need for systems to be able to compare and translate this information more intelligently. Stoter (2006) recommended the employment and development of machine ontology using the Web Ontology Language (OWL). There is a growing body of research around the application of ontologies to model and interpret urban information (Teller, Billen et al. 2010). This work forms part of that larger international initiative toward open spatial data access; a key example is the UK Ordnance Survey's GeoSemantics work where a set of ontologies describing the built environment are available for download and development (Ordnance Survey, 2010).

\section{THE AUSTRALIAN CONTEXT}

Sydney is facing the challenge of managing a pressing level of population growth combined with a tightly constrained geographic footprint. As such, planners and designers need to design better, denser urban environments to maximise economic, environmental and social benefits. For the purposes of demonstration, we have been focussing on five different government resources:

1. Floorspace \& Employment Survey (FSES)

2. Building Sustainability Index (BASIX)

3. Industry Foundation Classes (Ifc)

4. Strata titles database, containing property ownership data, and

5. SEPP65: Planning policy to improve the design quality of residential development.

\section{DATA INTEGRATION USING ONTOLOGIES}

An ontological approach provides a way of effectively browsing and retrieving concepts, their properties, relationships, and even geometric/geospatial representations. However, effective tools to connect ontologies with the content of the data sources are required. For the purposes of our research, we have integrated selected tools under development by TONES (2010). 
Table 1: Semantic mapping across government resources and documents concerning the built environment.

\begin{tabular}{|c|c|c|c|c|c|}
\hline CONCEPT & FSES & BASIX & IFC SCHEMA & STRATA & SEPP65 \\
\hline SITE(s) & Site_2006 & tagcadastre & IfcSite & Address & Context, Streetscape \\
\hline BUILDING & Building_2006 & building_details & IfcBuilding & Firsthousenum & Built form \\
\hline STOREY & Floor_2006 & .storeys & IfcBuildingStorey & Floor_num & Scale: Bulk, height \\
\hline SPACE & SpaceUnits_2006 & Dwelling details & IfcSpace, IfcZone & Lot_unit_num & Units, Rooms \\
\hline PROJECT & Establishment & project_details & IfcProject & Strata Plan (SP) & Process \\
\hline PERSON & Tenant, Surveyor & Accredited & IfcPerson, & Owner, & Social dimensions, Density \\
\hline
\end{tabular}

\subsection{Data Integration Issue}

Urban management involves various groups, and their associated endeavours create detailed repositories of digital information in various forms. These are complex, massive, fast updating and diverse. One such dataset is the City of Sydney's Floor Space and Employment Survey (FSES). This repository is a 3D geospatial dataset comprising of site, building and space information and corresponding geometry. These homogenous spatial clusterings have strong parallel concepts described in the Ifc schema. The Ifc schema forms an exhaustive taxonomy describing a building in detail down to that of its components. As such, this verbose list of elements and concepts forms the base level for a 'bottom-up' approach. This serves as a foundation to attach corresponding nodes and concepts found in other datasets concerning the urban domain. A test of the UrbanIT framework is to map these concepts across these different datasets.

\subsection{Application of Ontologies}

The previous section illustrated a bottom-up approach, however, when dealing with the complexity of urban environments, distilling the important elements into a manageable set of entities helps focus the framework in a top-down way. When using ontologies to connect the distinct datasets, we can identify the common concepts and map their terms together. In this case, the concepts that were important for our application were simply sites, buildings, storeys, and spaces.

\subsection{Semantic Concept Mapping}

Semantic mappings are used to bring the data sources, classes and properties of the ontologies together to assist users on data retrieval. For instance, the schematic 'IfcBuilding' can be mapped to the similar concept described in the FSES, listed at 'Building_2006'. Similarly, 'IfcSite' maps directly to 'Site_2006' and so on. Once these concepts are connected, we can map out how these elements relate to each other: for instance, "The Building sits on a Site".

Table 1 illustrates the common concepts spanning our different data and information sources, and lists the corresponding concepts identifying name from each data source (See section 4). Sometimes there is a direct connection between concepts, as with Ifc and FSES, but at other times inconsistencies, ambiguities and indirect references occur. This is mainly due to the fact that different data has been structured and collected in different contexts for different purposes. When the ontological layer spans across these sources, it has the capacity to homogenise them.

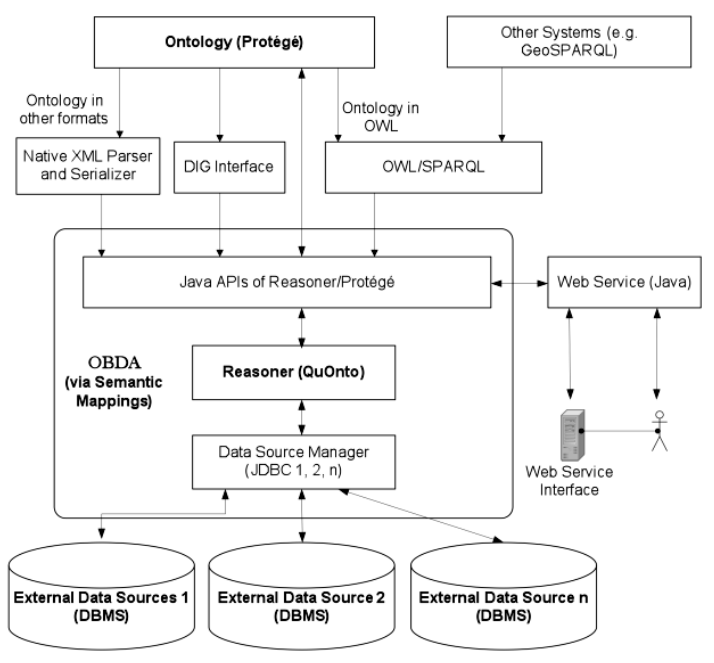

Figure 1: Framework Diagram.

\subsubsection{OBDA}

Ontology-Based Data Access (OBDA) is an area of research in which the goal is to provide access to data in heterogeneous sources through a semantic layer formed by ontology (Calvanese et al, 2009, DIG-OBDA, 2010). The main contribution of this 
research is to provide an end-to-end system to build a semantic layer for integration, to classify and reason by an OBDA-enabled reasoner and retrieve data through mapping. Another added value of OBDA, which applies particularly to the domain of urban planning, is that constraints expressed by the ontology allow users to overcome incompleteness that is present in the complex and fluid data captured from urban processes.

The OBDA Protégé plugin is developed by 'Knowledge Representation meets Databases' (KRDB). It provides complete views to relational database management systems (RDBMS), a mapping editing and testing environment, classification and reasoning built-in, and evaluated at the mapping phase and SPARQL (Protocol +RDF Query Language) queries reasoned by OBDA-enabled reasoners.

\subsubsection{QuOnto}

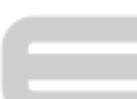

QuOnto is a reasoner that supports an OBDAenabled ontology. QuOnto is able to use an RDBMS as a repository for mappings between the data (Poggi et al. 2008).

\subsubsection{Architecture}

Figure 1 presents an architecture of an application driven by Protégé, OBDA and QuOnto.

\subsection{High Order Reasoning for Data Extraction}

Mapping connections in well-structured datasets is relatively straight forward, but the real strength of semantic techniques comes into play when semistructured, messy or otherwise disaggregated data is being interrogated. SEPP 65 is a (text-based) government policy document prescribing a set of guidelines to improve the design quality of residential apartment buildings. The document deals with objective and quantitative measures, such as the bulk and height, but also covers the qualitative and sometimes subjective aspects to urban developments. For instance, the policy states that:

"Good design responds and contributes to its context. Context can be defined as the key natural and built features of an area.

Responding to context involves identifying the desirable elements of a location's current character or, in the case of precincts undergoing a transition, the desired future character as stated in planning and design policies. New buildings will thereby contribute to the quality and identity of the area."

(State Environmental Planning Policy No 65Design Quality Of Residential Flat Development - Reg 9)

These elements and interrelationships can be documented logically and systematised so that the framework can carry out reasoning and logical testing. For instance, the building's context can be deduced as 'everything but the building, within an area, location or precinct'. It also can encompass time-based concepts such as 'precincts undergoing a transition'. At this point of development OpenCalais is being trialled to automatically mark up government documents such as SEPP65 for integration by the UrbanIT framework.

\subsection{Information Leverage \& Integration}

Ontologies provide data access by presenting many different channels: these could be a web portal, knowledge acquisition system or an object-oriented /relational database. For an end-user (e.g. an urban planner, decision maker or an urban information modeller), there is no need to be concerned about which channels are employed and what the connection is to each channel. Real-time, automatic processing and reasoning is handled transparently, so that the application acts as a one-stop experience to provide as much information as the user requires, through a service-oriented approach. This can be extended to an inter-organisational scale, to better provide support specific to advanced metropolitan strategic planning. This enhances inter-connectivity by fostering horizontal connections through open, unified and user-defined channels. This creates an opportunity for the development of a whole suite of new computer tools that can undertake multiple analyses at an urban level.

Figure 2 shows how energy use data might be retrieved and visualised from the BASIX database. The cooler colours (blue and green) reflect places that might have energy efficient Air-conditioners fitted, while the red spaces are less energy efficient. The surrounding buildings are coded by the FSES data to show actual context.

At the time of writing, the UrbanIT project is working closely with KRDB on more effective integration through VDB (Virtual Database, 2010) and retrieval by reasoning with incomplete/incompletely specified/missing data (Calvanese and Giacomo, 2008), both of which are 


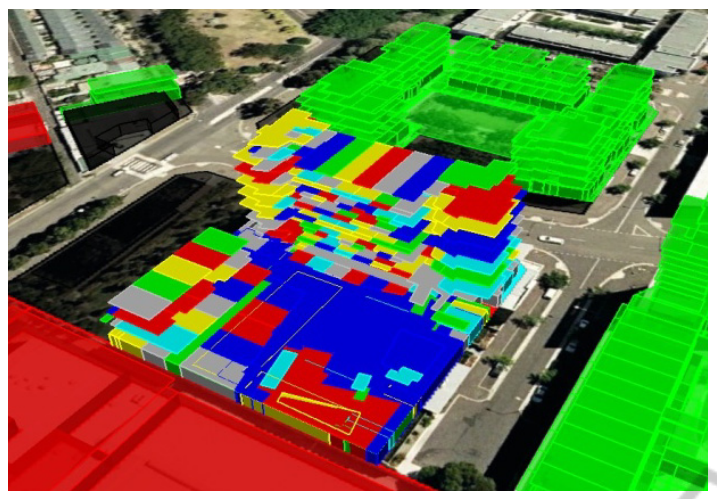

Figure 2: Query result illustrating possible energy ratings of air-conditioners.

poignant tools to meet the demands of improving the accessibility and usefulness of existing urban data repositories held by government.

\section{CONCLUSIONS}

These urban modelling exercises have indicated that the adoption of an ontological approach is valuable as it can assist in solving semi-structured urban problems. As a powerful modelling and integration tool, an ontology can be applied to the fundamental data layers, through to the higher layers where endusers are needing to make sense of otherwise scattered information, and make decisions about special urban challenges. A critical component of this workflow is OBDA. This enables 'vertical drilling' down through the 'horizontal' layers, which plays a central role in bringing heterogeneous and autonomous geographic and building information together to support urban decision making.

\section{ACKNOWLEDGEMENTS}

The UrbanIT project has been funded by the Australian Research Council (ARC) and undertaken in partnership with City of Sydney, NSW Department of Planning and Landcom. We are grateful for the support from Mariano RodríguezMuro and Diego Calvanese of the KRDB research group at the Free University of Bozen Bolzano in Italy by providing OBDA plugin and the QuOnto reasoner.

\section{REFERENCES}

Berners-Lee, T., J. Hendler, et al. (2001). "The Semantic Web." Scientific American May.

Calvanese, D. and G. De Giacomo (2008); Reasoning for Ontology Engineering and Usage, Part 4: Integrating Data into ontologies. Full day tutorial at the 7 th International Semantic Web Conference, Oct. 27, 2008, Karlsruhe, Germany.

Calvanese, D. et al (2009); Ontologies and Databases: The DL-Lite Approach, S. Tessaris et al. (Eds.): Reasoning Web 2009, LNCS 5689, p.255-356, 2009.

DIG-OBDA (2010); DIG1.1-OBDA, an OBDA extension

to the DIG1.1 interface, last accessed at http://obda.inf.unibz.it/dig-11-obda/extension-menu/ introduction/introduction.html

Gruber, T. (2009). Ontology. The Encyclopedia of Database Systems. L. Liu and M. T. Özsu, SpringerVerlag.

Katranuschkov, P., A. Gehre, et al. (2003). "An ontology framework to access IFC model data." ITcon 8: 413437.

Ordnance

\section{Survey}

(2010);

http:// www.ordnancesurvey.co.uk/oswebsite/ontology/ last accessed 27 July 2010

Poggi, A., Rodriguez-Muro, M and Ruzzi, M. (2008); Ontology-based database access with DIG-MASTRO and the OBDA Plugin for Protege. In: the proc. Of the $4^{\text {th }}$ International Workshop on OWL: Experiences and Directions (OWLED 2008 DC).

Protégé (2010); http://protege.stanford.edu/, last accessed July 27, 2010.

Stoter, J. E., Lemmens, R.L.G., Köbben, B.J. and Bakker, N. J. (2006). Semantic data integration in a multiple representation environment. Joint ISPRS working groups II/3 and II/6 workshop on multiple representation and interoperability of spatial data, Hannover.

Teller, J., R. Billen, et al. (2010). "Editorial - Bringing Urban Ontologies into Practice." ITcon 15(Special Issue Bringing urban ontologies into practice): 108110.

TONES (2010); http://www.tonesproject.org/ last accessed July 272010

Uschold, M. and M. Gruninger (2004). "Ontologies and semantics for seamless connectivity." SIGMOD Rec. 33(4): 58-64.

Virtual Database (2010); accessed at http://jboss.org/teiid/ basics/virtualdatabases.html 